\title{
A simple population model with a stochastic carrying capacity
}

\author{
C. Anderson $^{\mathrm{a}}$, Z. Jovanoski ${ }^{\mathrm{a}}$, I. N. Towers ${ }^{\mathrm{a}}$ and H. S. Sidhu $^{\mathrm{a}}$ \\ ${ }^{a}$ School of Physical, Environmental and Mathematical Sciences, UNSW Canberra, Australia.
} Email: c.anderson@adfa.edu.au

\begin{abstract}
Many ecosystems are subject to external perturbations such as pollution, land clearing and sudden shocks to their environment. However, most current models used do not take the changing environment into consideration. In cases where the changes in the environment are taken into account this is usually done by specifying some time-dependent function for the carrying capacity that reflects the observed behaviour of the changing environment.

Here we employ an alternative approach where the carrying capacity, a proxy for the state of the environment, is used as a state variable in the governing equations. Thereby, any changes to the environment can be naturally reflected in the survival, movement and competition of the species within the ecosystem.

In this paper a simple ecosystem consisting of a single species and its variable environment is studied. Specifically, a logistic population model that incorporates a stochastic carrying capacity is investigated. The carrying capacity is treated as a state variable and is described by a stochastic differential equation. The statistical properties of the carrying capacity and the population are analysed using the Monte Carlo method giving; the expected time evolution of the population and its variance, the probability distribution of the population and the mean-time to extinction.
\end{abstract}

Keywords: Logistic equation, Ornstein-Uhlenbeck process, stochastic differential equation, Monte Carlo simulation, carrying capacity 


\section{INTRODUCTION}

Verhulst's well known logistic model includes the concept of a 'saturation level' or carrying capacity, a value that represents the maximum population that an environment can sustain (Brauer and Catillo-Chàvez, 2001). The logistic equation is written as

$$
\frac{d N_{t}}{d t}=r N_{t}\left(1-\frac{N_{t}}{K}\right),
$$

where $N_{t} \equiv N(t)$ is the population at time $t, r$ is the intrinsic growth rate and $K$ is the carrying capacity.

This simple model has been adapted for over a century, some of which are detailed in Tsoularis and Wallace (2002). The carrying capacity, often assumed to be a constant (Oppel et al., 2014), can change due to exogenic and endogenic processes (Cohen, 1995). Models where the carrying capacity is treated as a function of time to describe changing environmental conditions can be found in Cushing (1977) and Coleman (1979).

The simplest approach to modelling a changing environment is by specifying the carrying capacity as a timedependent function (Safuan et al., 2013a). However, this approach is limiting, as it does not allow for the environment to be shaped by the interdependent relationships between the resources and the population that consumes them (del Monte-Luna et al., 2004). This issue was mitigated in a series of papers, Safuan et al. (2011, 2012, 2013b, 2014), models were developed that treated the carrying capacity as a state-variable coupled to the population model.

The variations to the logistic model mentioned thus far have their applications, however, there are many external environmental factors like; fire, drought, floods, resource contamination, etc that need to be accounted for. By adding stochasticity to the model, it is possible to account for the effects of such perturbations on the population that deterministic models ignore.

How stochasticity is added to population models is a modelling issue. Usually, environmental fluctuations are modelled by adding noise to the 'crowding term' (the reciprocal of the carrying capacity), the nonlinear term of (1), leading to (Kloeden and Platen, 1992; Méndez et al., 2010)

$$
d N_{t}=r N_{t}\left(1-\frac{N_{t}}{K}\right) d t+r \sigma N_{t}^{2} d W_{t}
$$

Here $W_{t}$ is a standard Weiner process with $\mathbf{E}\left[W_{t}\right]=0, \operatorname{Var}\left(W_{t}\right)=t$ and the noise intensity is $\sigma$.

\section{THE MODEL}

An alternate approach was proposed by Safuan et al. (2011). The approach is based on treating the carrying capacity as a state variable, that is, governed by a separate equation that describes the changes in the carrying capacity. A simple stochastic extension of the Safuan et al. (2011) model is

$$
\begin{aligned}
& \frac{d N_{t}}{d t}=r N_{t}\left(1-\frac{N_{t}}{K_{t}}\right), \quad N_{t=0}=N_{0} \\
& d K_{t}=-\gamma\left(K_{t}-k_{1}\right) d t+\sigma d W_{t}, \quad K_{t=0}=K_{0}
\end{aligned}
$$

The population, $N_{t}$, is still modelled by a logistic equation but now coupled to the carrying capacity, $K_{t}$, which in turn is modelled by a stochastic differential equation. Equation (4) consists of a deterministic and a stochastic term. In the absence of random variations, $\sigma=0,(3)$ and (4) can be solved exactly (Safuan et al. (2013a)). It represents a model for the growth of bacteria under an occlusion of the skin.

Equation (4) is commonly known as the Ornstein-Uhlenbeck equation (OU), a stochastic process that is stationary, Markovian and Gaussian (Uhlenbeck and Ornstein, 1930). The OU is 'mean-reverting' (drifts towards its long-term mean $k_{1}$ ). Figure 1 shows several realisations (paths or trajectories) of $K_{t}$ with different parameters. From the top plot the 'mean reverting' nature of OU can be seen: starting with different initial conditions $K_{0}$ all realisations approach $k_{1}$. How fast realisations of $K_{t}$ approach $k_{1}$ depends on $\gamma$. It places a constraint on how large the random variations can get. Large variations, either above or below $k_{1}$ are pulled back to $k_{1}$. The bottom plot shows realisation of $K_{t}$ for different noise intensities, $\sigma=1,0.5,0.1$. In particular it depicts a realisation that reaches $K_{t}=0$ in finite time when $\sigma$ is large, in a relative sense to other parameters. If the situation arises such that $K_{t}=0$, then the environment can no longer sustain the population leading to 

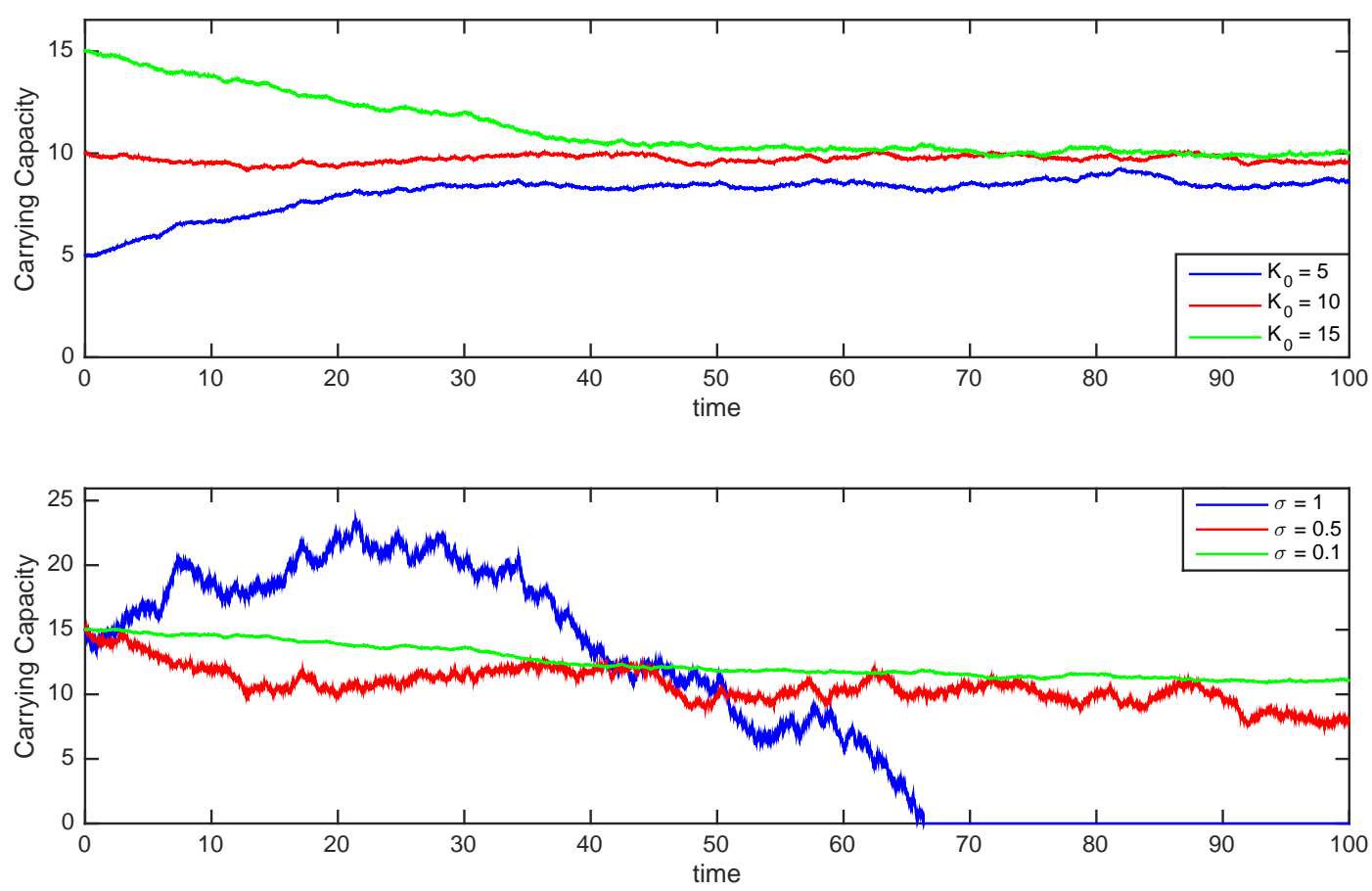

Figure 1. Three different realisations of $K_{t}$ with parameters (top) $\gamma=0.03$ and $\sigma=0.1$, (bottom) $K_{0}=15$ and $\gamma=0.01$. For both cases $k_{1}=10$.

extinction. From a conservationist perspective, predicting extinction times are very important (Foley, 1994). As (4) is independent of $N_{t}$ it can be solved separately, giving the well known solution of the OU equation

$$
K_{t}=k_{1}+\left(K_{0}-k_{1}\right) e^{(-\gamma t)}+\sigma \int_{0}^{t} e^{\gamma(s-t)} d W_{s}
$$

Substituting (5) into (3) leads to

$$
\frac{d N_{t}}{d t}=r N_{t}\left(1-\frac{N_{t}}{k_{1}+\left(K_{0}-k_{1}\right) e^{(-\gamma t)}+\sigma \int_{0}^{t} e^{\gamma(s-t)} d W_{s}}\right), \quad N_{t=0}=N_{0} .
$$

The exact solution of (6) is currently unknown, the numerical solution is discussed in the next section.

\section{EXPECTED SOLUTION PATH}

According to (5), the distribution of $K_{t}$ (conditioned on $K_{0}$ ) is normal, $K_{t} \sim N\left(\mu_{t}, \sigma_{t}^{2}\right)$, with (conditional) mean and variance

$$
\begin{aligned}
\mu_{t} & =\mathbf{E}\left[K_{t}\right]=k_{1}+\left(K_{0}-k_{1}\right) e^{-\gamma t}, \\
\sigma_{t}^{2} & =\operatorname{Var}\left(K_{t}\right)=\frac{\sigma^{2}}{2 \gamma}\left(1-e^{-2 \gamma t}\right) .
\end{aligned}
$$

However, from an ecological perspective, we require that $K_{t}>0$, therefore the exact conditional probability distribution for $K_{t}$ is (Feller, 1996)

$$
\mathbb{P}\left(K_{t}, t \mid K_{0}\right)=\frac{1}{\sqrt{2 \pi \sigma_{t}^{2}}}\left(e^{-\frac{\left(K_{t}-\mu_{t}\right)^{2}}{2 \sigma_{t}^{2}}}-e^{-\frac{\left(K_{t}+\mu_{t}\right)^{2}}{2 \sigma_{t}^{2}}}\right) .
$$



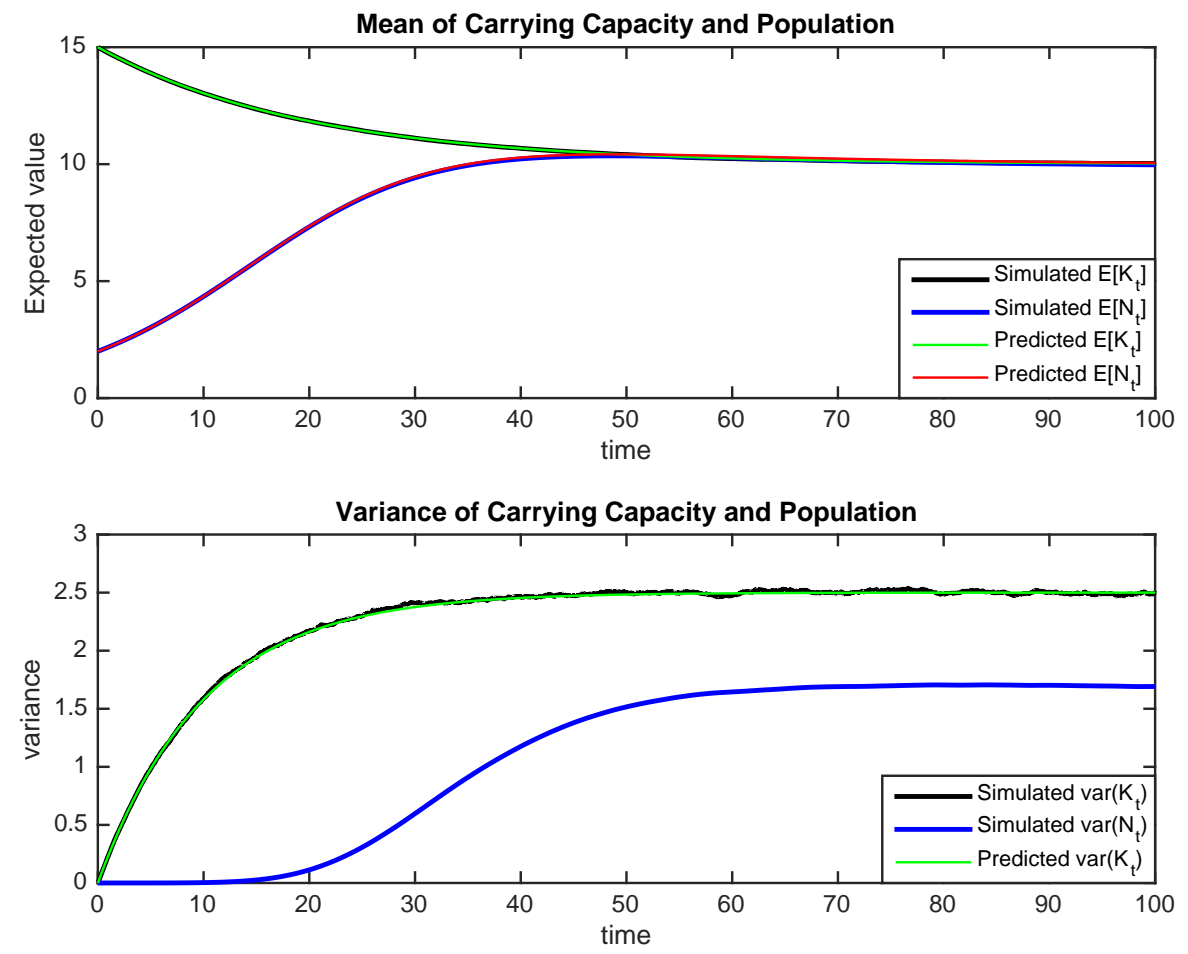

Figure 2. The mean and variance of the carrying capacity and the population. The plot shows both the numerically simulated values and the analytical model. Here $K_{0}=15, k_{1}=10, N_{0}=2, r=0.1, \gamma=0.05$ and $\sigma=0.5$.

Note that $\mathbb{P}\left(K_{t}=0, t \mid K_{0}\right)=0$ constitutes an absorbing boundary condition; when the carrying capacity reaches zero it remains there. Ecologically this condition represents the collapse of the environment thus leading to the extinction of the population.

Knowing the properties of $K_{t}$, a Monte Carlo approach was employed to determine the distribution of $N_{t}$ and its mean and variance. This was achieved by constructing 40,000 realisations of OU (5). For each realisation, (6) was solved numerically using Euler's method with a step size of $\Delta t=0.01$. This size of $\Delta t$ was a good compromise between accuracy and computational effort. The mean and variance of $K_{t}$ and $N_{t}$ are plotted in Figure 2.

On both plots, the simulated $K_{t}$ is plotted in black, the simulated $N_{t}$ is plotted in blue, for the initial conditions $K_{0}=15$ and $N_{0}=2$. The top plot shows the (conditional) mean of $K_{t}$ which coincides with the predicted mean given by (7). It is possible to write (6) formally as

$$
N_{t}=\frac{e^{r t}}{\frac{1}{N_{0}}+\int_{0}^{t} \frac{r e^{r s}}{K_{s}} d s}
$$

An exact algebraic expression for $\mathbf{E}\left[N_{t}\right]$ is not possible. An approximate expression for the (conditional) mean of $N_{t}$ is obtained by replacing $K_{s}$ in (10) with $\mathbf{E}\left[K_{s}\right]$

$$
\mathbf{E}\left[N_{t}\right] \approx \frac{e^{r t}}{\frac{1}{N_{0}}+\int_{0}^{t} \frac{r e^{r s}}{\mathbf{E}\left[K_{s}\right]} d s}
$$

The predicted mean for $N_{t}$ is also shown in the plot (red). Apart from the early transient behaviour the means of $K_{t}$ and $N_{t}$ both approach $k_{1}$, independent of the initial conditions. 

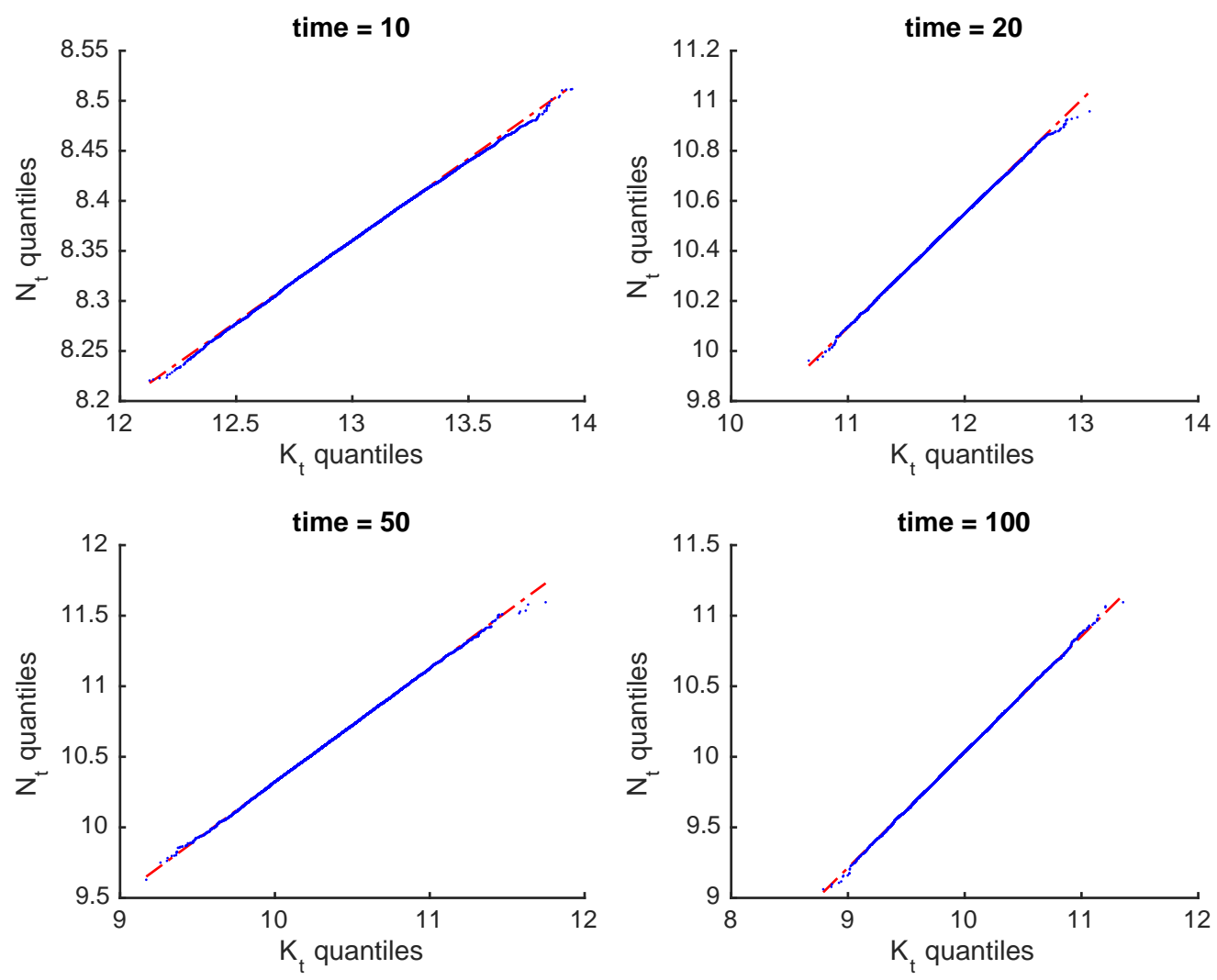

Figure 3. Quantile-quantile plots at different times of the population $\left(N_{t}\right)$ against the carrying capacity $\left(K_{t}\right)$. The parameters are: $K_{0}=15, N_{0}=5, k_{1}=10, r=0.1, \gamma=0.05$ and $\sigma=0.1$.

Similarly, in the bottom plot, the (conditional) variance of $K_{t}$ matches up with the theoretical expression given by (8). No simple expression for the variance of $N_{t}$ could be found. We note that $\operatorname{Var}\left(N_{t}\right)<\operatorname{Var}\left(K_{t}\right)$ for all time $t$. However, beyond some time, $t \gg 1 / \gamma$, each asymptotically converges to some constant, but different, value.

A quantile-quantile plot (qq-plot) of the simulated values of $N_{t}$ against $K_{t}$ is shown in Figure 3. It demonstrates that the distributions are similar and since the slope of the line is less than one it further indicates that $\operatorname{Var}\left(N_{t}\right)<\operatorname{Var}\left(K_{t}\right)$, as mentioned previously.

\section{DistribUtion OF EXTINCTION TIMES}

As alluded to previously we require $K_{t}>0$ for all $t$. However as can be seen from Figure 1 there is a realisation for which $K_{t}$ reaches zero. According to (4) this is quite feasible depending on the relative size of $\gamma$ and $\sigma$, for fixed $k_{1}$ and $K_{0}$. On the characteristic time scale of $1 / \gamma$ the dynamics of $K_{t}$ is predominantly governed by the Weiner process, $W(t)$, and if $\sigma$ is large then it is possible for $K_{t}$ to reach zero. The instant $K_{t}=0$, it remains there and the environment can no longer sustain the population, resulting in its extinction.

The probability of the environment collapsing by time $t$ is $1-S\left(t \mid K_{0}\right)$ where $S\left(t \mid K_{0}\right)$ is the survival function

$$
S\left(t \mid K_{0}\right)=\int_{0}^{\infty} \mathbb{P}\left(K_{t}, t \mid K_{0}\right) d K_{t} .
$$

The distribution of the first-hitting (extinction) time represents the probability density for which $K_{t}$ has collapsed by time $t$. It is defined by $f\left(t \mid K_{0}\right)=-S^{\prime}\left(t \mid K_{0}\right)$. The distribution is (Wang and Uhlenbeck, 1945) 


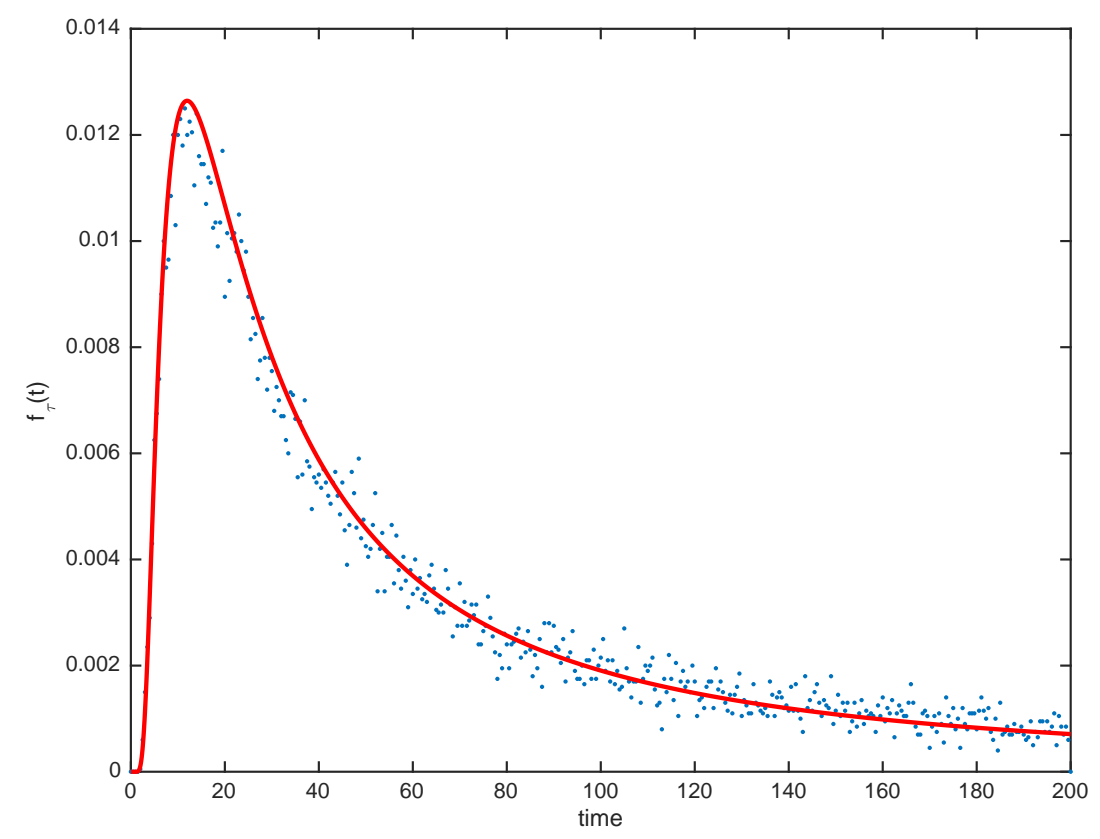

Figure 4. Plot showing both the theoretical and numerical probability distribution function of the first-hitting time for $K_{t}$ with $K_{0}=12, k_{1}=10, r=0.5, \gamma=0.01$ and $\sigma=2$.

$$
f(t)=\frac{2 e^{\left(-\frac{\mu_{t}^{2}}{2 \sigma_{t}^{2}}\right)}}{\sqrt{2 \pi \sigma_{t}^{2}}} \cdot\left(\frac{\mu_{t} \sigma_{t}^{\prime}}{\sigma_{t}}-\mu_{t}^{\prime}\right),
$$

where $\mu_{t}$ and $\sigma_{t}^{2}$ are given by (7) and (8), respectively. Here, $\mu_{t}^{\prime}$ and $\sigma_{t}^{\prime}$ are the derivatives of the mean and variance. Figure 4 is a plot of the probability density function for the first-hitting times. The Monte Carlo simulations are shown as dots (blue) and the line is the theoretical probability density function given by (12). It is clear that (12) is a good approximation. Furthermore, extensive numerical simulations indicated that the first-passage times for $N_{t}$ may also be approximated by those of $K_{t}$.

\section{Conclusion}

In this paper, the carrying capacity is considered as a proxy variable for the state of the environment in which a species is assumed to reside in, such as bacteria under an occlusion of the skin (Safuan et al., 2011, 2013a). In this context the stochastic environment is approximately described by the OU process. The 'mean reverting' aspect of the OU process is reflected in the fact that the skin (body) can 'self-regulate' thus skin conditions do not greatly depart from normal (Safuan et al., 2011). We have investigated the implications of coupling the OU process, as a proxy for random fluctuations in environmental conditions, to the logistic equation (3). The exact probability function for $K_{t}$ was found. The qq-plots indicated that $N_{t}$ has a similar distribution to $K_{t}$. Beyond the early transient period, the means are the same and the variance is constant, albeit smaller for $N_{t}$.

These observations can be explained with reference to (3) and (4). No matter how complicated (4) may appear and as long as it is independent of $N_{t}$ then in principle it can be solved for $K_{t}$. From the differential equation for $N_{t}$, when $K_{t}$ is higher than $N_{t}, N_{t}$ will increase toward $K_{t}$ and when $K_{t}$ is lower it will decrease toward $K_{t}$. This implies that the population $N_{t}$ will always pursue the carrying capacity $K_{t}$. In the special case where $r$ is large this means that the rate of change of $N_{t}$ will be large in magnitude so that it follows $K_{t}$ more closely. For this reason, the fluctuations of $N_{t}$ will always be smaller than $K_{t}$, $\operatorname{Var}\left(N_{t}\right)<\operatorname{Var}\left(K_{t}\right)$.

In the extreme case where $r \rightarrow \infty$ we have $N_{t}=K_{t}$ so that the distribution of $N_{t}$ will be the same as the 
Anderson et al., A simple population model with a stochastic carrying capacity

distribution of $K_{t}$. In this case only do we have $\operatorname{Var}\left(N_{t}\right)=\operatorname{Var}\left(K_{t}\right)$.

The interesting case occurs when $r$ is small, in which the distribution of $N_{t}$ may no longer be similar to $K_{t}$. It could be approximated by simulation and use of kernel density estimators.

A full analysis requires the construction and solution of the associated Fokker-Planck equation to (3) and (4) with appropriate (absorbing) boundary conditions $\left(K_{t}=0\right.$ or $\left.N_{t}=0\right)$. Even in this case, the analysis would be formidable. It may be possible to make progress in the cases for which the intrinsic growth rate, $r$, is very large or when $0<r \ll 1$. This is currently under investigation.

\section{REFERENCES}

Brauer, F. and C. Catillo-Chàvez (2001). Mathematical Models in Population Biology and Epidemiology. New York: Springer.

Cohen, J. E. (1995). Population growth and earth's human carrying capacity. Science 269, 341-346.

Coleman, B. D. (1979). Nonautonomous logistic equation as models of the adjustment of populations to environmental change. Math. Biosci. 45, 159-173.

Cushing, J. M. (1977). Periodic time-dependent predator-prey systems. Siam J. Appl. Math. 32, 82-95.

del Monte-Luna, P., B. W. Brook, M. J. Zetina-Rejón, and V. H. Cruz-Escalona (2004). The carrying capacity of ecosystems. Global Ecology and Biogeography 13, 485-495.

Feller, W. (1996). An Introduction to Probability Theory and Its Applications. Wiley Series in Probability and Mathematical Statistics, Vol II. New York: John Wiley and Sons, Inc.

Foley, P. (1994). Predicting extinction times from environmental stochasticity and carrying capacity. Conservation Biology 8, 124-137.

Kloeden, P. E. and E. Platen (1992). Numerical Solutions of Stochastic Differential Equations. Berlin: Springer-Verlag.

Méndez, V., I. Liopis, D. Campos, and W. Horsthemke (2010). Extinction conditions for isolated populations affected environmental stochasticity. Theoretical Population Biology 77, 250-256.

Oppel, S., H. Hilton, N. Ratcliffe, C. Fenton, J. Daley, G. Gray, V. J., and D. Gibbons (2014). Assessing population viability while accounting for demographic and environmental uncertainty. Ecology 95, 18091818.

Safuan, H. M., Z. Jovanoski, I. N. Towers, and H. S. Sidhu (2011, December). A simple model for the total microbial biomass under occlusion of healthy skin. In 19th International Congress on Modelling and Simulation, Perth, Australia, pp. 733-739.

Safuan, H. M., Z. Jovanoski, I. N. Towers, and H. S. Sidhu (2012). Coupled logistic carrying capacity model. ANZIAM J. 54, C172-C184.

Safuan, H. M., Z. Jovanoski, I. N. Towers, and H. S. Sidhu (2013a). Exact solution of a non-autonomous logistic population model. Ecological Modelling 251, 99-102.

Safuan, H. M., Z. Jovanoski, I. N. Towers, and H. S. Sidhu (2013b). Impacts of biotic resource enrichment on predator-prey population. Bull. Math. Biol. 75, 1798-1812.

Safuan, H. M., Z. Jovanoski, I. N. Towers, and H. S. Sidhu (2014). A two-species predator-prey model in an environment enriched by a biotic resource. ANZIAM J. 54, C768-C787.

Tsoularis, A. and J. Wallace (2002). Analysis of logistic growth models. Math. Biosci 179, 21-55.

Uhlenbeck, G. E. and L. S. Ornstein (1930). On the theory of brownian motion. Phys. Rev. 36, 823-841.

Wang, M. C. and G. E. Uhlenbeck (1945). On the theory of the brownian motion ii. Review of Modern Physics 17, 323-342. 